- Palmer dental notation can readily be reproduced in word-processed documents by the use of bespoke font characters.

- The same method also has scope for use in other common software applications that support the use of multiple fonts.

- Users of Microsoft Windows XP ${ }^{\mathrm{TM}}$ can create the required specialised characters without the need for additional imported fonts or font creation software.

- Electronic transmission and viewing of documents is also feasible, provided that the recipient computer is suitably configured, or the sender embeds the font within the document.

\title{
The Palmer notation system and its use with personal computer applications
}

\author{
J. W. Ferguson ${ }^{1}$
}

The arguments for and against the Palmer dental notation system are briefly discussed, including the perceived difficulty of reproducing this on personal computers. Some technical solutions to the problems encountered in everyday Windows-based programs are outlined, with suggestions as to possible future applications.

\section{INTRODUCTION}

There has been much debate in the columns of this and other journals regarding the merits or otherwise of the various tooth notation systems currently in use. Two methods which will be familiar to most UK dentists are the Palmer and the FDI systems. Several authors have come out strongly in favour of the FDI system ${ }^{1-3}$ and have encouraged the international dental community to adopt this as the sole method. However, this does not seem to have gained widespread acceptance, at least within the United Kingdom. Blinkhorn et $a l^{4}$ found that only one UK dental school actually instructed its students to use FDI notation as standard but, despite this, most of the practitioners referring patients to that institute still seemed to prefer the Palmer method.

One advantage of Palmer notation is that it can produce a very graphical image, akin to a 'map' of the dentition; tooth transpositions or edentulous spaces can easily be depicted if desired. It would also be feasible to introduce additional alphabetic characters or other symbols, for example to denote supernumerary teeth or bridge pontics, to which a purely numerical

1*Orthodontic Department, Walsall Manor Hospital, Moat Road, Walsall, West Midlands, WS2 9PS

${ }^{*}$ Correspondence to: Dr J. W. Ferguson

Email:jim.ferguson@walsallhospitals.nhs.uk

\section{Refereed Paper}

Received 19.01.04; Accepted 10.01.05

doi: 10.1038/sj.bdj.4812303

() British Dental Journal 2005; 198: 551-553 method such as the FDI system does not lend itself easily. There is a perceived problem, however, with reproducing Palmer notation on computers. Indeed, in view of these difficulties, the British Dental Journal itself requested a change to the tooth notation system to be used by prospective authors. ${ }^{5}$ Nevertheless, as stated above, it still seems to be popular amongst UK practitioners, and thus it would seem logical to try and resolve the technical issues surrounding computer replication, particularly word processing. Some possible solutions for incorporating Palmer notation into word processed documents and other applications are thus described below.

\section{Word processing}

One solution was proposed by Lewis, ${ }^{6}$ using the Microsoft Word equation editor function. This was primarily to deal with the problem of lower tooth characters which require an overline, the upper teeth simply being underlined and right/left separated thus. It required several keystrokes, although it was possible to automate the process to a large extent by the use of a macro function.

Another way to generate the overline is simply to include this as part of a bordered table inserted within the document. In Microsoft Word, this can also be saved as an autotext entry, a blank table being entered where required and the necessary teeth entered in the appropriate table cells from the keyboard. The table needs to be so designed so that the visible borders (that is, those that will actually print) are restricted to the appropriate cell boundaries. The major disadvantage is that it is not possible to incorporate a table on the same line as normal text, therefore careful composition of the document is required to make the text flow. An example of how this might be used is shown in Figure 1 .

The use of bespoke fonts is potentially the most versatile solution. McCormack ${ }^{7}$ originally described the use of dedicated fonts which could be used in dentistry. He demonstrated how specialised font creation and modification software (Fontographer version 4.1; Macromedia Inc., 600 Townsend Street, San Francisco, CA 94103: www.macromedia.com) could be used to produce images of teeth, implants, etc which could then be incorporated into documents. A logical extension of this was to produce a font in which the individual tooth numbers and letters could be depicted in Palmer format, with the relevant lines placed over/under and right/left of the number or letter itself. ${ }^{8}$ The present author has been using such a font (designed by John McCormack) for several years now. A further refinement, which the present author has recently developed, is to create characters without left/right dividers, but to store the latter as separate symbols. This enables continuous charting of a given quadrant, and is the format in which most people would choose to write Palmer notation longhand. Examples of the text that can be produced with specialised fonts are shown in Figure 2. 


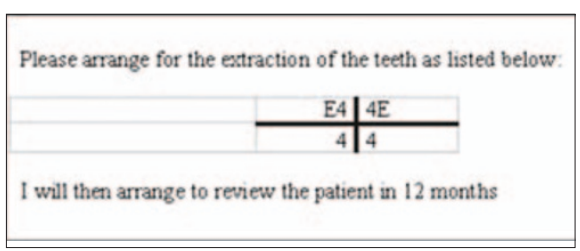

Fig. 1 A table inserted into a document to facilitate Palmer-style notation. The thick lines (which have been exaggerated for clarity) would appear in print, the thinner lines representing those table cell borders which are only visible on screen.

As mentioned above, creation of such fonts normally requires the use of specific software. Those using the Microsoft Windows XP operating system, however, can create their own Palmer notation characters with a little-known function called Private Character Editor (PCE) which is already included with the software. A description of the procedure is given in the appendix. However, whereas a freestanding font generated with proprietary software can be distributed and installed on any personal computer, PCE characters cannot be exported. This is of no consequence if the user simply wishes to produce hard-copy output, but has implications for the electronic transmission of documents (see below).

For a typist wishing to enter Palmer notation, there are two options. One is to manually switch fonts when a section of document requiring such notation is reached, the other is to store the characters as autotext entries which are assigned to buttons on customised toolbars (see Fig. 3 and appendix). Clicking on the button then inserts the character, in the correct font, with automatic reversion to the default font selected for the document when normal keyboard entry is resumed.

\section{Other Microsoft applications}

The above method can also be used for other products in the Microsoft range, including PowerPoint, Publisher, Frontpage and Wordpad, which also support the use of multiple fonts within the same document. The main difference is that it is not possible to create toolbars for rapid character entry as with Microsoft Word. Nevertheless, as the vast majority of users who may wish to use fonts in other programs

The teeth requiring extraction are $E \mathrm{~A} \mid 4 \mathrm{E}$ and $\overline{4} \overline{4}$

The teeth requiring extraction are E] 4$]\lfloor\underline{4}[E$ and $\overline{4} \sqrt{4}$

Fig. 2 Text produced using specialised font characters. The upper line shows the effect using a font with tooth numbers/letters simply underlined or overlined, separate characters being used to divide left and right quadrants. On the lower line, each individual tooth is depicted by a unique character.

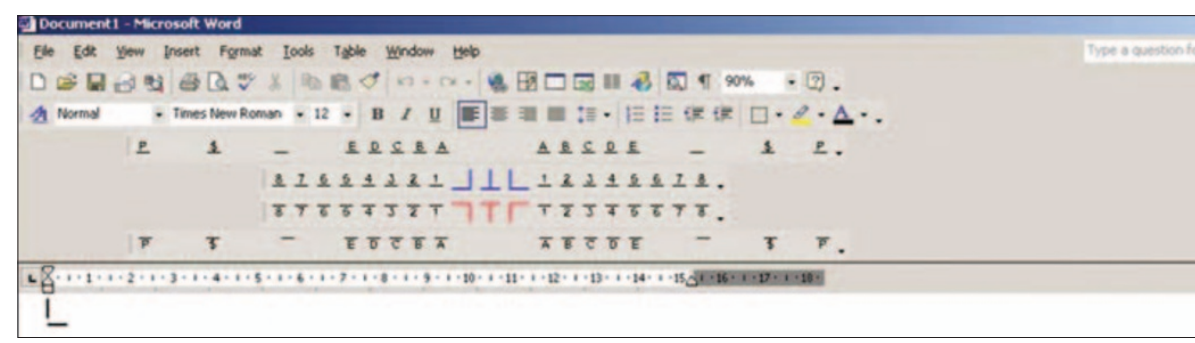

Fig. 3 Microsoft Word screen set up with customised toolbars to permit continuous charting, using the character set illustrated in Figure 6 . Clicking on the button text inserts the symbol into the document. Four toolbars are used, arranged on screen to mimic the Palmer grid. The toolbars for the deciduous teeth (which also include suggested additional symbols for supernumerary teeth, pontics and spaces within the dentition) have been placed above and below those for the permanent teeth. The button images for the quadrant starters, finishers and dividers are coloured for easier identification. The corresponding tooth symbols to left and right of the dividers do, in fact, insert identical autotext entries; the layout chosen is simply to make the screen more intuitive.

would also have Word installed, it is a simple matter to generate the required text, copy and paste it into the desired application. With PowerPoint, all of the text may default to that selected for new text objects within that document, but the relevant sections can be selected and manually altered to the required font.

Microsoft Access (a relational database program) is a special case: a detailed discussion of the possible variations in database design, and how best to record the dentition, is beyond the scope of this article. However, printed outputs (reports) may be required which will have the same visual format as word-processed documents. The simplest option is, of course, to create a separate data field for each quadrant, in which case standard alphanumeric characters, corresponding to the Palmer numbers or letters for the required teeth, can be entered in that field direct from the keyboard. Input forms and reports are then set up with the relevant fields oriented correctly from the perspective of the user, with quadrant dividing lines constructed on these forms or reports using the toolbox function. This will produce an output comparable to a bordered table in Microsoft Word. There is a specific problem if one wishes to include descriptive text along with dental notation within the same database field, as each field can only display a single font. The solution is to use a font which contains regular text and punctuation in addition to the specific dental characters. The author uses both methods within his hospital clinical database.

\section{Electronic transmission of documents}

The ideal situation is for the recipient to have the relevant font installed on their computer, which will allow any document to be viewed and edited if required. Plain email texts can also contain multiple fonts, which will often display correctly on the receiving computer if the above conditions are satisfied although, in the author's experience, this is not always reliable. For potential recipients who are unlikely to have the font installed, electronic transmission of the font file together with the message is perfectly feasible, and installation is straightforward. Microsoft Word and PowerPoint have an option to embed fonts within the document (see appendix), thus allowing them to be viewed even if the recipient's computer is not set up appropriately. Once again, in the author's experience, this works reliably in Word 2002, and also Word 97, but not in earlier versions. The above options are not applicable if the characters have been created using the Private Character Editor function in Windows $\mathrm{XP}$, but if the receiving computer is also running Windows XP it is possible to configure it with identical characters. Editing of documents with embedded fonts is not possible, but may not actually be necessary or desirable (for instance, for an electronic referral). A further option, which would be appropriate for small documents, is to send them as graphic files; this format would, in any case, be required for radiographs and clinical photographs. Files can be created either by scanning or using the 'Print Screen' function; if the 'Full Screen' option is selected from Word's 'View' menu, all menus and toolbars are hidden and not included with the screen clip. The graphic can be copied, pasted into another document, and also saved as a separate file. Windows XP can save these clips as .JPEG files, which are of a much more manageable size than standard bitmaps. Finally, web-based documents or journals could incorporate Palmer notation if desired, as it would be perfectly feasible to provide for a simultaneous download of the font from the publisher's website.

\section{Other potential applications}

These could include databases for electronic patient records, which are likely to become the standard of the future, and developers of such systems could consider incorporating Palmer-type fonts as part of any specialised functionality developed for the dental field. Such databases may also 
Fig. 4 The PCE code selection grid, showing part of a character set charting and also insertion of unique characters for each individual tooth. Hidden rows are displayed using the vertical scrol arrow. The unique (hexadecimal) reference code for each character is determined by its position at the row/column intersection. created to allow both continuous

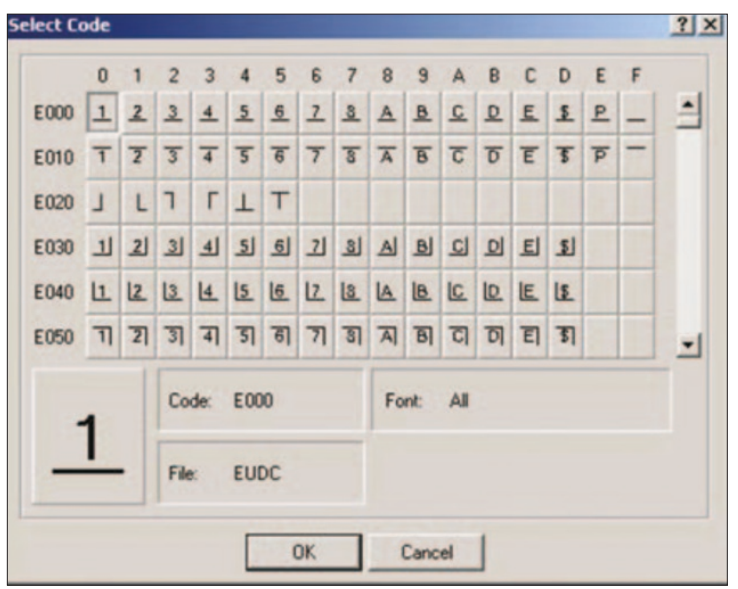

incorporate clinical coding systems, but if reference to specific teeth is required it would be far more appropriate to include these in a separate field and not as part of the diagnosis or procedure code itself. For example, using the OPCS-4 codes (employed in the author's hospital) for descriptions of dental procedures, it would clearly be unworkable to devise separate codes for all possible combinations that might be covered by even one of the existing codes (F104, extraction of multiple teeth).

\section{Summary}

The fact that previous research ${ }^{4}$ has suggested that Palmer notation is still the preferred system amongst UK graduates argues favourably for its continued use, especially as the technical problems in reproducing it in word-processing and other software packages are surmountable. Electronic communication between practitioners would be facilitated by the widespread dissemination of the required specialised fonts within the dental community, and an agreed standardisation of the character codes for those wish-

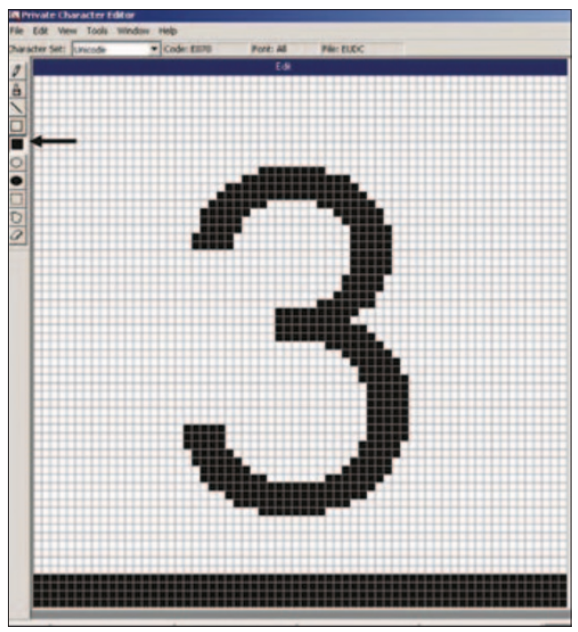

Fig. 5 The PCE editing grid; the arrow shows the filled rectangle tool, which has been selected and dragged over the requisite number of pixels to insert the underline. The number ' 3 ' has first been pasted into the grid by using the 'Copy Character' function, which is accessed via the 'Edit' menu. ing to use the existing font editing functionality within Windows XP.

\section{Appendix}

Creating dental symbol characters using Windows Windows 'Help' files (enter 'PCE' in the Search box which should display 'Using Special Characters': click on 'Related Topics' and follow the links.)

1. Click on 'Start', then 'Run'.

2. Type in 'eudcedit' (without the quotation marks). 3. This opens the PCE window with the 'Select Code' screen displayed (Fig. 4).

4. Double-click on one of the squares in the selection grid which will open the bitmap editing window for that character space.

5. Copy the outline for the required alphanumeric character by selecting 'Copy Character' from the 'Edit' menu.

6. Then, using the filled rectangle tool (black square) from the toolbar on the left hand side of the screen, create the required over/underlines or left/right divider lines as required by dragging over the required pixels. A line thickness of four pixels should give an adequate on-screen display (Fig. 5).

7. Any mistakes can be rectified by using the eraser tool, at the foot of the toolbar.

8. Select 'Save Character' from the 'Edit' menu, or 'Next Code' from the 'View' menu.

9. To view the complete character set, or select another character code which does not immediately follow or precede the current character in sequence, choose 'Select Code' from the 'Edit Menu' again.

Fig. 6 Character map window showing private characters created with PCE. Select 'All Fonts (Private Characters)' from the font list box to display this character set. XP Private Character Editor (PCE)

Below is a brief description of how to use this function; full instructions can be found in the

\section{Inserting characters into document}

Characters created using PCE can be found under 'All Fonts (Private Characters)' in the character map font list box (Fig. 6). They cannot be inserted directly from the keyboard. They can be selected and copied into a new blank Word document, from which individual autotext entries can be created and assigned to custom toolbars. Instructions for accessing the character map, creating autotext entries and custom toolbars can be found in the Windows or Word help files. Autotext entries can also be created from installable fonts, although in this case it is also possible to arrange the encoding layout such that they can be entered by logicallyarranged keystrokes (eg unshifted numbers and letters for lower teeth, shift key plus same characters for upper teeth, etc.)

\section{Embedding True-Type fonts}

1. From the Word or Powerpoint 'Tools' menu select 'Options'.

2. Click the 'Save' tab.

3. Under 'Save Options', check the box captioned 'Embed True-Type Fonts'.

4. Note that in Powerpoint the embedding option applies to the current document only.

The author particularly wishes to thank John McCormack for his invaluable help and assistance, and to acknowledge his original idea of using specialised fonts for dental iconography. Thanks are also due to Sharon Bradley for her assistance with drafting the manuscript and providing an end-user perspective with regard to integrating specialised fonts into word-processing applications.

1. Peck S, Peck L. A time for change of tooth numbering system. J Dent Educ 1993; 57: 643-647.

2. Sandham J A. The FDI two-digit system of designating teeth. Int Dent J 1983; 33: 390-392.

3. Elderton R J. Keeping up to date with tooth notation. Br Dent J 1989; 166: 55-56.

4. Blinkhorn A S, Choi C L, Paget H E. An investigation into the use of the FDI tooth notation system by dental schools in the UK. Eur J Dent Educ 1998; 2 39-41.

5. Grace M. Dental notation. Br Dent J 2000; 188: 229.

6. Lewis D H. Dental notation (Letter). Br Dent J 2000; 188: 230-231

7. McCormack J. Iconographic dental typography. A dental character font for computer graphics. $\mathrm{Br}$ Dent J 1991; 170: 417-420.

8. McCormack J. (Personal communication.) 1998. 